(2) Open Access Full Text Article

\title{
Success of ${ }^{125}$ I-Seed Treatment in Vulvar Squamous-Cell Carcinoma with Aplastic Anemia: A Case Report
}

This article was published in the following Dove Press journal: OncoTargets and Therapy

\author{
Qingtao Ni (iD) \\ Chi $\mathrm{Pan}^{2}$ \\ Qing Guo' \\ Peng Wang' \\ Guangzhi Sun' \\ Shujun $\mathrm{Xiao}^{3}$ \\ Shengbin Dai' \\ 'Department of Oncology, Jiangsu \\ Taizhou People's Hospital, Taizhou \\ 225300, People's Republic of China; \\ ${ }^{2}$ Department of General Surgery, Jiangsu \\ Taizhou People's Hospital, Taizhou \\ 225300, People's Republic of China; \\ ${ }^{3}$ Department of General Practice, Jiangsu \\ Taizhou People's Hospital, Taizhou \\ 225300, People's Republic of China
}

Correspondence: Shujun Xiao Department of General Practice, Jiangsu Taizhou People's Hospital, Taizhou 225300, People's Republic of China Tel +86 139-526I-9404

Email 78340454@qq.com

Shengbin Dai

Department of Oncology, Jiangsu Taizhou People's Hospital, Taizhou 225300,

People's Republic of China

Tel +86 I35-1515-57/7

Email135I5I557I7@I63.com

\begin{abstract}
Vulvar squamous-cell carcinoma (SCC) is a rare disease that occurs mainly in postmenopausal women. Chemo/radiotherapy with or without surgery is the most important modality for treatment of advanced vulvar cancer. A case of vulvar SCC with aplastic anemia was treated using ${ }^{125}$ I seeds in our department, because surgery and chemotherapy were not possible due to low platelets, leaving radiotherapy as the lone therapeutic option. ${ }^{125}$ I seeds present an alternative option for treatment of patients with vulvar SCC and local relapse with lymph-node metastasis following previous radiotherapy.
\end{abstract}

Keywords: vulvar squamous-cell carcinoma, I-125 seeds, aplastic anemia, ${ }^{125} \mathrm{I}$

\section{Introduction}

Vulvar cancer is the fourth-most frequent type of gynecological cancer, accounting for approximately $5 \%$ of all female genital tract malignancies. ${ }^{1}$ According to data from the American Cancer Society, 6,120 cases of vulvar cancer were newly diagnosed and 1,350 women died from this disease in the US in $2020 .^{2}$ The most common histological type of vulvar cancer is squamous-cell carcinoma (SCC), which accounts for around $90 \%$ of cases. ${ }^{3}$ Surgery is the main treatment for vulvar cancer, ${ }^{4}$ with radical resection of the vulva and bilateral inguinal lymphadenectomy commonly applied surgical modalities. ${ }^{5}$ Current standard surgical treatments frequently lead to unsatisfactory outcomes, particularly in patients with node-positive disease. ${ }^{6}$ Further, chemo/radiotherapy, with or without surgery is the most important treatment modality for advanced vulvar cancer. ${ }^{7}$ Advanced vulvar SCC has limited treatment options, and recurrence is often lethal. ${ }^{8}$ Despite the number of treatment options, patients continue to be afflicted by posttreatment side effects, including substantial morbidity, lymphedema, sexual and psychological dysfunction, and wound-healing disorders. ${ }^{9}$ Moreover, up to $40 \%$ of patients with earlystage vulvar SCC develop recurrence within 10 years. ${ }^{10}$ Unfortunately, not all patients are suitable for surgery and chemotherapy, such as those with aplastic anemia (AA), a type of acquired bone marrow-failure syndrome. ${ }^{11}$ AA is an immunomediated disease caused by the destruction of hematopoietic cells, resulting in pancytopenia. ${ }^{12}$

Implantation of iodine $125\left({ }^{125} \mathrm{I}\right)$ seeds into tumors is a targeted-radiotherapy method. Under the guidance of ultrasound and other imaging techniques, ${ }^{125} \mathrm{I}$ seeds, which emit low-energy gamma rays, are directly implanted into tumors to kill the 
malignant tissue. As the dose intensity of ${ }^{125} \mathrm{I}$ is inversely proportional to the square of distance, the cumulative dose in normal tissue around the target tumor is minimized. ${ }^{13}$ This local treatment is safe and effective, and can provide long-term local control of tumors. ${ }^{14}$ Adjunctive radiotherapy, such as ${ }^{125} \mathrm{I}$ seeds, significantly lowers the risk of recurrence at the site of the primary tumor relative to patients treated without radiotherapy. ${ }^{15}$ For some patients with recurrence or localized metastasis after surgery, the use of ${ }^{125}$ I seeds has obvious advantages. ${ }^{125}$ I seeds combined with chemotherapy and other systemic therapy can also kill distant metastatic lesions, with clear effects on tumor treatment. In addition, this approach can also be used to supplement conventional radiotherapy and as a means of coordinated treatment to achieve better therapeutic effects. ${ }^{16}$

Here, we report a case of vulvar SCC in a patient with AA who was treated using ${ }^{125}$ I seeds in our department in recent years.

\section{Case Report}

Here, we present the case of a 50-year-old premenopausal woman who had developed painless, slowly growing masses accompanied by irregular bleeding under the skin of the vulva in August 2016. The masses were located in the left labium majus of the vulva, without palpable inguinal lymph nodes bilaterally. One was $3 \times 3 \mathrm{~cm}$ and the other $2 \times 2 \mathrm{~cm}$. No ulceration was observed. Laboratory tests (hormone panel, and gynecological and breast cancer markers) were within normal limits. Pathological examination of a vulva biopsy on August 19, 2016 showed moderately and poorly differentiated SCC (Figure 1). A total-body computed-tomography scan and whole-body bone imaging, performed to determine disease stage, were negative for distant metastases.

The patient had a medical history of AA of $>20$ years, and had taken folic acid, androgens, and cyclosporine to control the disease. The specific dose of medication was unknown. However, the drugs were ineffective. Subsequently, she was treated with regular blood transfusions, with platelet counts $\leq 10 \times 10^{9} / \mathrm{L}$. Further, she had a history of type 2 diabetes of $>4$ years.

Due to the low platelet count of the patient because of AA, surgery and chemotherapy were not possible. Local radiotherapy with DT72GY/36FX was performed from August 24, 2016 using noncoplanar electron-beam perpendicular irradiation with conventional linear accelerator on vulvar masses covered with $0.5 \mathrm{~mm}$ wet gauze, and the patient placed in the lithotomy position at a collimator angle of $90^{\circ}$ to the linear accelerator. A significant regression of the masses in
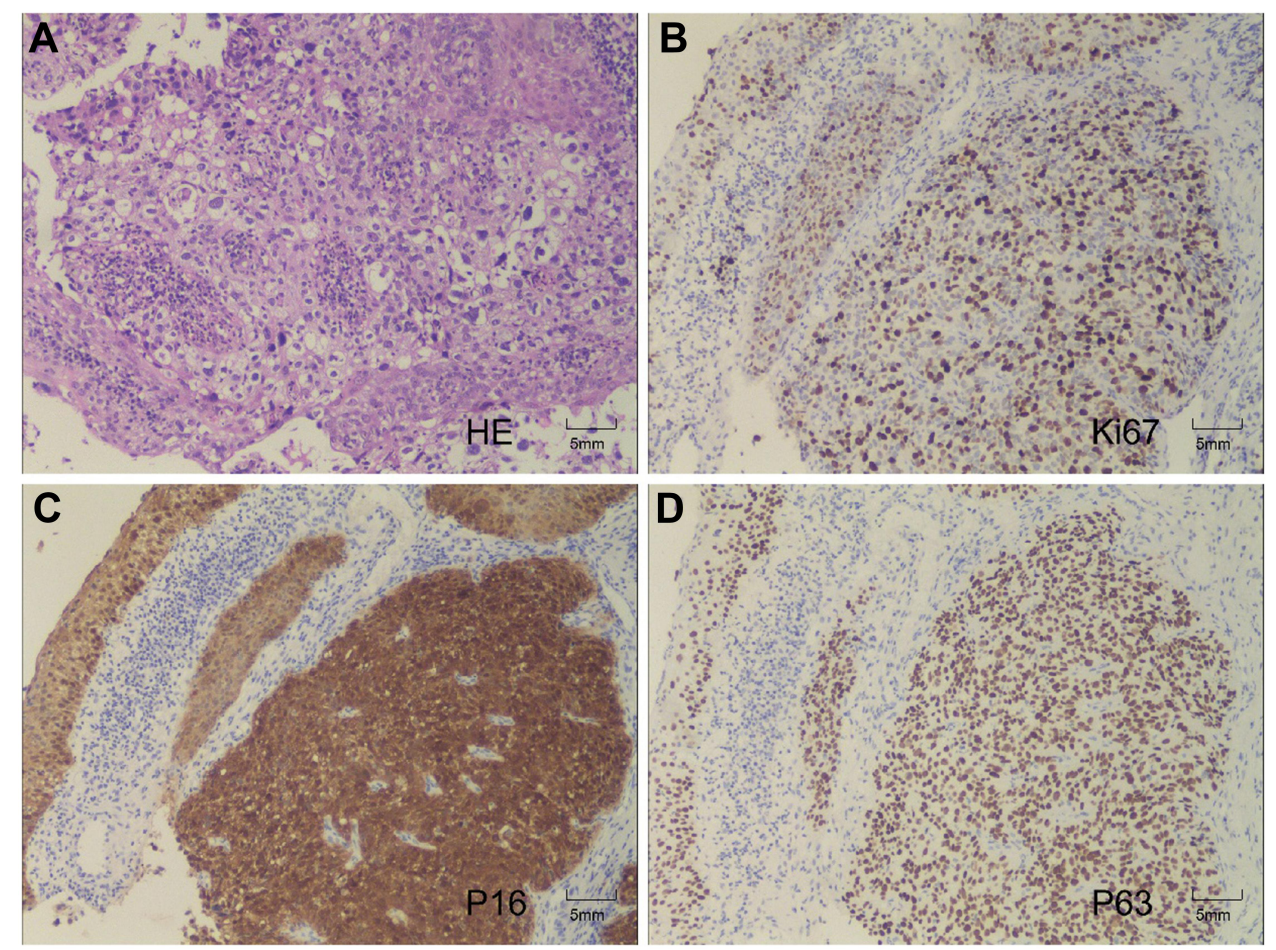

Figure I Histopathologic specimen. (A) H\&E staining, 100×. (B) Ki67 staining, 100×. (C) pl6 staining, 100×. (D) p63 staining, 100×. 
the vulva was found on subsequent physical examination; however, she had grade II radiation injury of the skin, which is common and unavoidable, despite the use of radioprotective agents. During treatment, platelets and red blood cells were supplemented by occasional component blood transfusion.

In August 2018, the patient palpated a hard mass in the left inguinal groin, with poor mobility and no tenderness, which had gradually increased in the previous 2 months. On pelvic MRI, a mass of approximately $37 \times 35 \mathrm{~mm}$ was observed in the left inguinal region (Figure 2). Due to coagulation disorder resulting from AA, a biopsy was not performed. A diagnosis of metastasis in the left inguinal lymph node was considered, based on MRI. Radiotherapy of the left inguinal groin may cause lymphatic reflux disturbance; therefore, the patient was treated with $35^{125}$ I seeds in the left inguinal node under the guidance of ultrasound on October 26, 2018. The biologically effective dose of an
${ }^{125} \mathrm{I}$ seed is almost $100 \mathrm{~Gy}$. The ${ }^{125} \mathrm{I}$ seeds were mainly implanted in the center of the mass at a distance of $1 \mathrm{~cm}$ or more from the edge to reduce damage to the skin. On November 18, 2018, the left inguinal mass was approximately $33 \times 33 \mathrm{~mm}$ on MRI (Figure 3 ). Therefore, the patient was again treated with eight ${ }^{125} \mathrm{I}$ seeds in the left inguinal node as a supplementary local dose on January 18, 2019.

On February 22, 2019, the patient had a mass in the vulva. A $21 \times 16 \mathrm{~mm}$ mass and a nodular shadow of approximately $9 \times 8 \mathrm{~mm}$ were detected in the left inguinal region on pelvic MRI (Figure 4), indicating that the mass in the groin was receding and the particle implantation was effective. Therefore, 5 days later, ${ }^{125} \mathrm{I}$ seeds were implanted into the base of the new masses in the vulva and left inguinal region. The superficial mass in the vulva gradually subsided soon after seed implantation. At the same time, skin ulceration was still appearing.
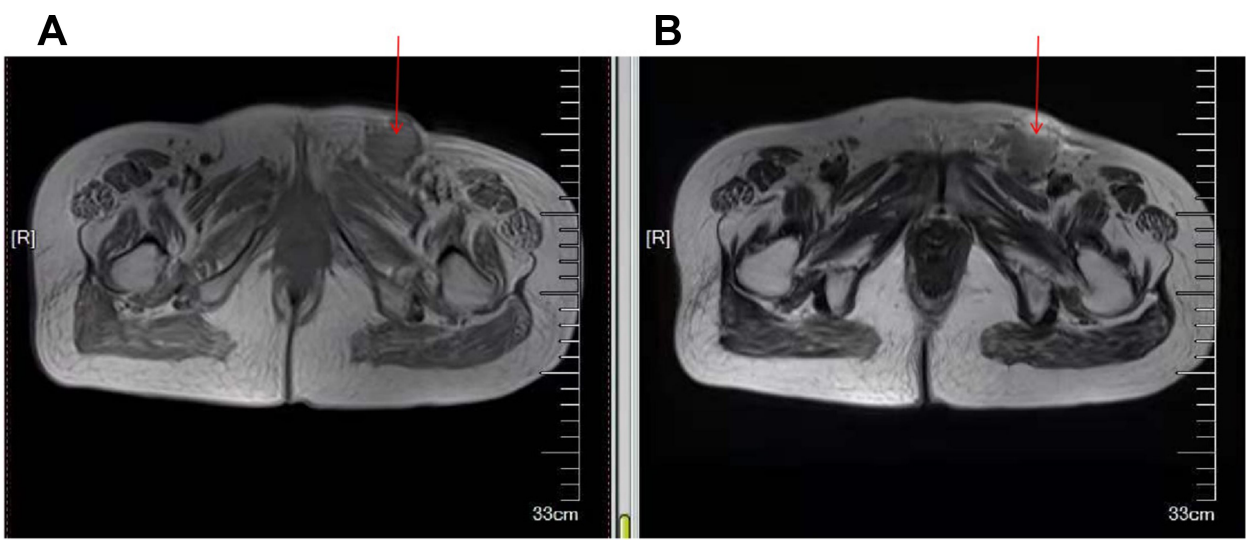

Figure 2 Mass of $37 \times 35 \mathrm{~mm}$ detected in the left inguinal region on pelvic MRI. (A) Low signal intensity of approximately $37 \times 35 \mathrm{~mm}$ detected in the left inguinal region on $T_{1}$ -weighted imaging (arrow). (B) High signal intensity of approximately $37 \times 35 \mathrm{~mm}$ detected in the left inguinal region on $T_{2}$-weighted imaging (arrow).

A

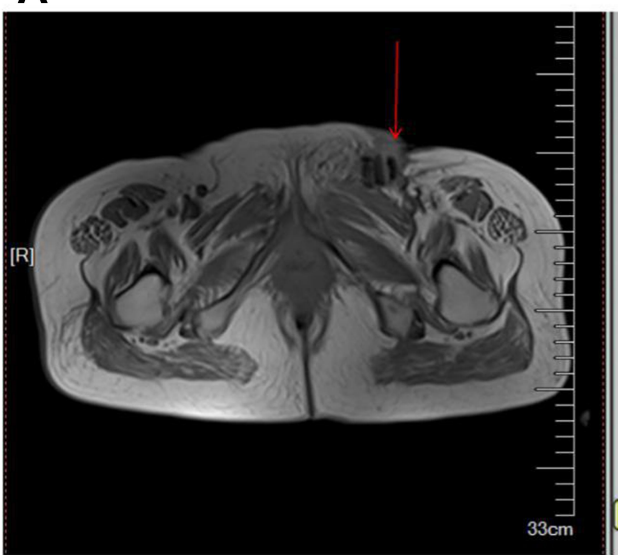

B

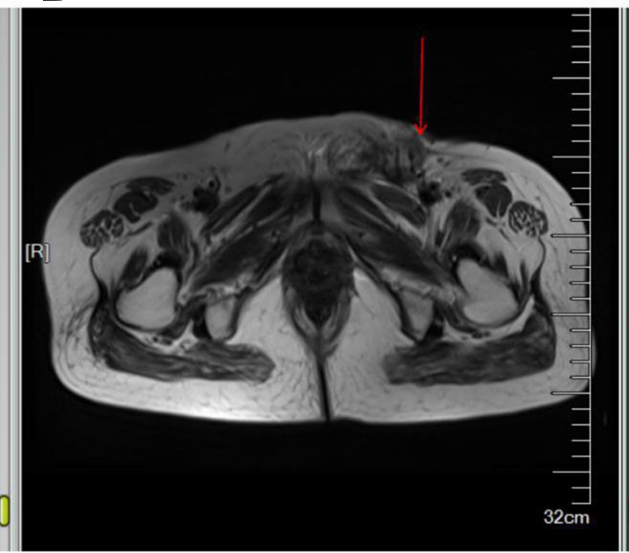

Figure 3 Mass of $33 \times 33 \mathrm{~mm}$ detected in the left inguinal region on pelvic MRI. (A) Low signal intensity of approximately $33 \times 33 \mathrm{~mm}$ detected in the left inguinal region on $T_{1}$ -weighted imaging (arrow). (B) High signal intensity of approximately $33 \times 33 \mathrm{~mm}$ detected in the left inguinal region on $T_{2}$-weighted imaging (arrow). 


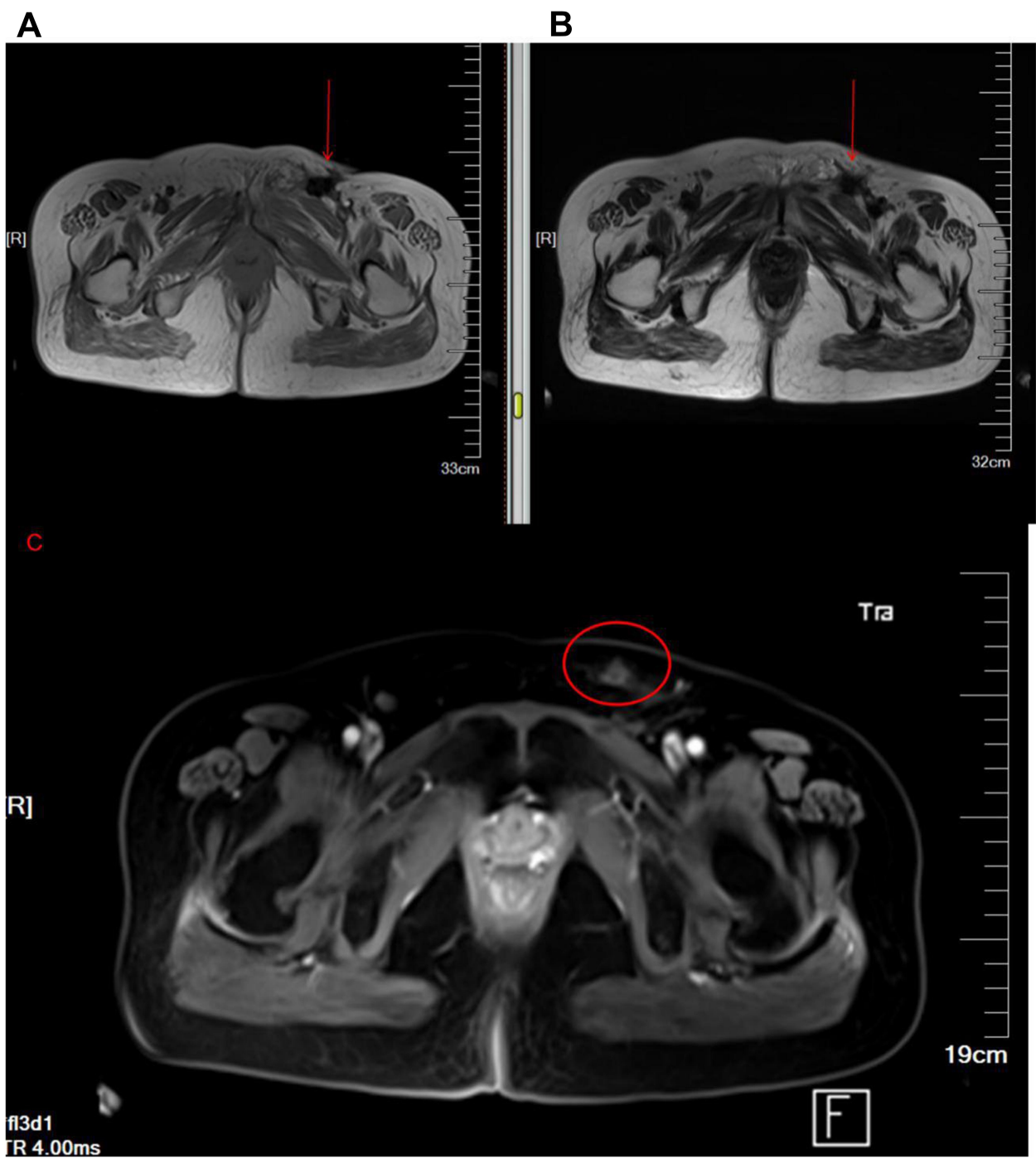

Figure 4 Mass of $21 \times 16 \mathrm{~mm}$ and a nodular shadow of approximately $9 \times 8 \mathrm{~mm}$ detected in the left inguinal region on pelvic MRI. (A) Low signal intensity of approximately $21 \times 16 \mathrm{~mm}$ detected in the left inguinal region on $T_{1}$-weighted imaging (arrow). (B) High signal intensity of approximately $21 \times 16 \mathrm{~mm}$ was detected in the left inguinal region on $T_{2}$-weighted imaging (arrow). (C) Mild enhancement of a nodular shadow $(9 \times 8 \mathrm{~mm})$ after injection of contrast-enhancing agent (circle).

Unfortunately, 2 months later, the patient developed two more palpable lumps: one of $3 \times 3 \mathrm{~cm}$ and the other $2 \times 2 \mathrm{~cm}$. Simultaneously, a $15 \times 6 \mathrm{~mm}$ mass was detected in the left inguinal region on ultrasound. Therefore, on May 10, 2019, the patient was treated with implantation of $60{ }^{125}$ I seeds in the base of the new lump and left inguinal region. After implantation, the mass completely disappeared again. Although the patient developed bleeding of the vulvar mass, it stopped in response to symptomatic treatment (wet wrap with $0.002 \%$ norepinephrine daily and $0.6 \mathrm{~g}$ ethylenediamine diaceturate intravenously guttae daily). The ulcers of the epidermis remained.

According to data collected at follow-up examinations performed between May 2019 and June 2019, the patient was able to conduct normal activities, and no new lesions were found. Unfortunately, the patient developed a cough and hemoptysis, and multiple metastatic lesions were found in the lungs on July 12, 2019. In August 2019, the patient received radiotherapy with DT45GY/15FX for the lesions in the lungs; however, the cough and hemoptysis were aggravated following DT27GY/9FX radiotherapy. The patient died on October 31, 2019.

\section{Discussion}

This was a unique case of vulvar SCC with AA in a 50year-old premenopausal woman. Vulvar cancer mainly occurs in older women, primarily those who are postmenopausal. ${ }^{17}$ The peak age of incidence of vulvar cancer has been reported as $>70$ years; however, the peak period for vulvar cancer among Chinese women is $<60$ 
years. ${ }^{18}$ The patient reported here was a middle-aged premenopausal woman with a history of AA. Surgical treatment is usually the first choice for patients with earlystage vulvar SCC, including radical vulvectomy with bilateral inguinofemoral lymphadenectomy as a treatment of choice for vulvar $\mathrm{SCC} ;{ }^{19}$ however, the substantial loss of tissue involved in such surgery results in a risk of postoperative complications. ${ }^{20}$ After consultation with several hospitals, it was concluded that surgery and chemotherapy would be intolerable for our patient, because of her history of AA. In this case, radiotherapy was the only option. Radiation therapy has been regarded as a highly effective treatment for patients with noninvasive and inoperable vulvar cancer, ${ }^{21}$ with recurrence rates of $20 \%$ and $35 \%$ for patients with stage III and IV disease, respectively. $^{22}$

Vulvar cancer lesions can directly infiltrate adjacent tissue and organs, such as the vagina, urethral orifice, and anus, or spread directly to the opposite side. Further, vulvar cancer can spread from its original site to locoregional nodes. The 5-year overall survival rate for patients with vulvar cancer without nodal metastasis is $>90 \%$, while that for patients with nodal metastasis is $<60 \%{ }^{23}$ Our patient was clinically assessed as lymph-node negative at first diagnosis. Unfortunately, she was unable to sustain prolonged disease-free survival, and experienced local relapse and nodal involvement 2 years after the first radiotherapy. Due to the risk of exceeding normal tissue tolerance, doses are often limited for patients with recurrence after previous radiotherapy. Nevertheless, brachytherapy, such as ${ }^{125}$ I seeds, allows dose escalation in such situations, which can provide a perfectly targeted and localized boost dose and potentially improve patient outcomes. $^{24}$

For ${ }^{125}$ I seeds, a physical dose of 100 Gy delivered at an initial dose rate of approximately $0.05 \mathrm{~Gy} /$ hour, is equivalent to $50 \mathrm{~Gy}$ of external-beam radiotherapy in fractions of $2 \mathrm{~Gy}^{25}$ Compared with conventional externalbeam radiotherapy, implantation of ${ }^{125} \mathrm{I}$ seeds involves a smaller irradiation dose, longer effect, and more accurate treatment positioning to achieve genuine image-guided radiotherapy and conformal radiotherapy. ${ }^{125}$ I seeds provide a high local dose effect to tumors, with a small radiation radius and minimal damage to surrounding normal tissue. The radiation dose from ${ }^{125}$ I seeds is inversely proportional to the square of distance, and the effective diameter of particle radiation is only $17 \mathrm{~mm} .^{26}$ Therefore,
${ }^{125}$ I seeds represent an excellent local treatment for a wellselected patient population. ${ }^{27}$

Following treatment with ${ }^{125} \mathrm{I}$ seeds, although the patient developed redness and swelling of the skin after particle implantation, the therapeutic effect was relatively good. To prevent the occurrence of skin symptoms, hyaluronic acid injection can be used to provide space between the tumor and the skin in patients with subdermal tumors, resulting in decreased doses to the skin during brachytherapy. $^{28}$

\section{Conclusion}

${ }^{125}$ I seeds represent an alternative therapeutic opportunity for patients with vulvar SCC who do not have the option of surgery and chemotherapy and for those with local relapse with lymph-node metastasis after previous radiotherapy.

\section{Ethics Statement}

This study was approved by the Human Ethics Review Committee of Jiangsu Taizhou People's Hospital.

\section{Consent to Publish}

Written informed consent to publish this report and the associated medical images was provided by the patient prior to her passing.

\section{Author Contributions}

All authors made a significant contribution to the work reported, whether in conception, study design, execution, acquisition of data, analysis and interpretation, or in all these areas, took part in drafting, revising, or critically reviewing the article, gave final approval to the version to be published, have agreed on the journal to which the article has been submitted, and agree to be accountable for all aspects of the work.

\section{Disclosure}

The authors report no conflicts of interest in this work.

\section{References}

1. Hecking T, Thiesler T, Schiller C, et al. Tumoral PD-L1 expression defines a subgroup of poor-prognosis vulvar carcinomas with non-viral etiology. Oncotarget. 2017;8:92890-92903. doi:10.18632/oncotarget. 21641

2. Siegel RL, Miller KD, Jemal A. Cancer statistics, 2020. CA Cancer J Clin. 2020;70:7-30. doi:10.3322/caac. 21590

3. Anastasi E, Gigli S, Ballesio L, et al. The complementary role of imaging and tumor biomarkers in gynecological cancers: an update of the literature. Asian Pac J Cancer Prev. 2018;19:309-317. 
4. Niu Y, Yin R, Wang D, et al. Clinical analysis of neoadjuvant chemotherapy in patients with advanced vulvar cancer: A STROBE-compliant article. Medicine. 2018;97:e11786. doi:10.10 97/MD.0000000000011786

5. Saner FA, Schotzau A, Mackay G, et al. Fibrin-thrombin sealant does not reduce lymphocele formation in patients with inguinofemoral lymphadenectomy for vulvar cancer. Cancer Manag Res. 2019;11:3575-3582. doi:10.2147/CMAR.S197143

6. Hockel M, Trott S, Dornhofer N, et al. Vulvar field resection based on ontogenetic cancer field theory for surgical treatment of vulvar carcinoma: a single-centre, single-group, prospective trial. Lancet Oncol. 2018;19:537-548. doi:10.1016/S1470-2045(18)30109-8

7. Zobec LH. Long term results of radiotherapy in vulvar cancer patients in slovenia between 1997-2004. Radiol Oncol. 2017;51: 447-454. doi:10.1515/raon-2017-0024

8. Clancy AA, Spaans JN, Weberpals JI. The forgotten woman's cancer: vulvar squamous cell carcinoma (VSCC) and a targeted approach to therapy. Ann Oncol. 2016;27:1696-1705. doi:10.1093/annonc/mdw242

9. Kortekaas KE, Santegoets SJ, Abdulrahman Z, et al. High numbers of activated helper $\mathrm{T}$ cells are associated with better clinical outcome in early stage vulvar cancer, irrespective of HPV or p53 status. J Immunother Cancer. 2019;7:236. doi:10.1186/s40425-019-0712-z

10. G N T, Pouwer AW, de Bock GH, et al. Prognostic factors for local recurrence of squamous cell carcinoma of the vulva: A systematic review. Gynecol Oncol. 2018;148:622-631. doi:10.1016/j.ygyno.20 17.11 .006

11. Zhang X, Liu L, Dou C, et al. PPAR gamma-regulated microRNA 199a-5p underlies bone marrow adiposity in aplastic anemia. Mol Ther Nucleic Acids. 2019;17:678-687. doi:10.1016/j.omtn.2019.07.005

12. Scheinberg P, Chen J. Aplastic anemia: what have we learned from animal models and from the clinic. Semin Hematol. 2013;50:156-164. doi:10.1053/j.seminhematol.2013.03.028

13. Ma X, Yang Z, Jiang S, et al. Hybrid optimization based on non-coplanar needles for brachytherapy dose planning. J Contemp Brachytherapy. 2019;11:267-279. doi:10.5114/jcb.2019.86167

14. Xiang Z, Li G, Liu Z, et al. 125 i brachytherapy in locally advanced nonsmall cell lung cancer after progression of concurrent radiochemotherapy. Medicine. 2015;94:e2249. doi:10.1097/MD.000 0000000002249

15. Vora GK, Demirci H, Marr B, et al. Advances in the management of conjunctival melanoma. Surv Ophthalmol. 2017;62:26-42. doi:10.10 16/j.survophthal.2016.06.001

16. Song Y, Chan MF, Burman C, et al. Comparison of two treatment approaches for prostate cancer: intensity-modulated radiation therapy combined with $125 \mathrm{I}$ seed-implant brachytherapy or $125 \mathrm{I}$ seed-implant brachytherapy alone. $J$ Appl Clin Med Phys. 2008;9:2283. doi:10.1120/jacmp.v9i2.2283
17. Peri M, Grassadonia A, Iezzi L, et al. A case of stage i vulvar squamous cell carcinoma with early relapse and rapid disease progression. Case Rep Oncol Med. 2019;2019:1018492.

18. Xiao X, Meng YB, Bai P, et al. Vulvar cancer in china: epidemiological features and risk analysis. $J$ Cancer. 2017;8:2950-2958. doi:10.7150/jca.20496

19. Jiang Y, Tian R, Yu S, et al. Clinical significance of galectin-7 in vulvar squamous cell carcinoma. Oncol Lett. 2015;10:3826-3831. doi:10.3892/ol.2015.3826

20. Dias AR Jr, Soares JM Jr, de Faria M, et al. Secondary healing strategy for difficult wound closure in invasive vulvar cancer: a pilot case-control study. Clinics. 2019;74:e1218. doi:10.6061/ clinics/2019/e1218

21. Saito T, Tabata T, Ikushima $H$, et al. Japan society of gynecologic oncology guidelines 2015 for the treatment of vulvar cancer and vaginal cancer. Int J Clin Oncol. 2018;23:201-234.

22. Sznurkowski JJ, Milczek T, Emerich J. Prognostic factors and a value of 2009 FIGO staging system in vulvar cancer. Arch Gynecol Obstet. 2013;287:1211-1218. doi:10.1007/s00404-012-2683-x

23. Edge SB, Compton CC. The American Joint Committee on Cancer: the 7th edition of the AJCC cancer staging manual and the future of TNM. Ann Surg Oncol. 2010;17:1471-1474. doi:10.1245/s10434010-0985-4

24. Brun T, Ferron G, Filleron T, et al. Experimental study of pelvic perioperative brachytherapy with iodine 125 seeds (I-125) in an animal model. J Contemp Brachytherapy. 2018;10:463-469. doi: $10.5114 /$ jcb. 2018.79470

25. Baltas D, Lymperopoulou G, Loffler E, et al. A radiobiological investigation on dose and dose rate for permanent implant brachytherapy of breast using $125 \mathrm{I}$ or $103 \mathrm{Pd}$ sources. Med Phys. 2010;37:2572-2586. doi:10.1118/1.3426027

26. Wang W, Liu Z, Zhu J, et al. Brachytherapy with iodine 125 seeds for bone metastases. J Cancer Res Ther. 2017;13:742-747. doi:10.4103/ jcrt.JCRT_399_17

27. Logghe P, Verlinde R, Bouttens F, et al. Long term outcome and side effects in patients receiving low-dose 1125 brachytherapy: a retrospective analysis. Int Braz J Urol. 2016;42:906-917. doi:10.1590/S1677-5538.IBJU.2015.0542

28. Yuan Y, Ying Z, Jianjian D, et al. Subdermal injection of hyaluronic acid to decrease skin toxicity from radiation delivered with low-doserate brachytherapy for cancer patients. J Contemp Brachytherapy. 2019;11:14-20. doi:10.5114/jcb.2019.82770

\section{Publish your work in this journal}

OncoTargets and Therapy is an international, peer-reviewed, open access journal focusing on the pathological basis of all cancers, potential targets for therapy and treatment protocols employed to improve the management of cancer patients. The journal also focuses on the impact of management programs and new therapeutic agents and protocols on patient perspectives such as quality of life, adherence and satisfaction. The manuscript management system is completely online and includes a very quick and fair peer-review system, which is all easy to use. Visit http://www.dovepress.com/ testimonials.php to read real quotes from published authors. 